\title{
Cataract Surgery with Introcular Lens Implantation in Fuchs' Heterochromic Cyclitis
}

\author{
C. M. JAKEMAN*, K. JORDAN $\dagger$, J. KEAST-BUTLER* ${ }^{*}$, S. PERRY \\ Cambridge and Ely
}

\begin{abstract}
Summary
The results of cataract extraction with posterior chamber lens implantation in twenty eyes with Fuchs' Heterochromic Cyclitis are reported. No serious operative complications were experienced. The visual outcome has been excellent in all but those eyes with coexistent glaucoma. The postoperative complications seem no more frequent than would be expected for the underlying condition.
\end{abstract}

Fuchs' heterochromic cyclitis (FHC) is not a common condition, with estimates of its incidence at between $1.5 \%{ }^{1}$ and $4.5 \%{ }^{2}$ of referred uveitis cases. However, its almost universal association with the development of cataract ${ }^{3,4}$ which often progresses to maturity and profound visual loss, together with the young age at presentation and predominantly unilateral nature, have important implications for management if the best visual outcome is to be achieved.

Reports of cataract extraction alone have shown a variable outcome. Some authors have suggested that surgery is uncomplicated and the visual prognosis good. ${ }^{5,6,7}$ Others have described complications at surgery and a poor visual outcome. ${ }^{4,8}$ In particular problems with post operative hyphaema and elevation of intra-ocular pressure have been described.

Recently attempts have been made to restore binocular useful vision by cataract extraction combined with primary implantation of an intra-ocular lens. ${ }^{9,10,11}$ The results have been encouraging with good visual outcome and few reported complications. However reports have combined the results of both intracapsular and extracapsular extraction and the use of anterior chamber, iris fixated or iridocapsular fixated intraocular lens.

The combination of glaucoma, ${ }^{4,12}$ iris hypoperfusion, ${ }^{13,14}$ iridocorneal angle vascularisation, ${ }^{8,15,16}$ and decreased corneal endothelial count,${ }^{14}$ would suggest, on theoretical grounds, potentially fewer complications from surgery combining extracapsular extraction with a posterior chamber lens implant.

We report the results of 20 cases of FHC with combined extracapsular cataract extraction and posterior chamber intraocular lens. The operative and postoperative complications and the longer term outcome are discussed.

\section{Materials and Methods}

The diagnosis of FHC was based on the finding of iris heterochromia associated with iris crypt blunting and atrophy, minimal anterior chamber reaction with only few cells and flare, scattered small white keratitic precipitates and absence of posterior synechiae. ${ }^{7,17}$ Vitreous and fundal examination was performed in all cases to exclude a diagnosis of intermediate or posterior uveitis.

From June 1983 at Ely and Addenbrookes

Departments: *Department of Ophthalmology, Addenbrookes Hospital, Cambridge; †Department of Ophthalmology, West Suffolk Hospital (formerly RAF (H), Ely); ‡Department of Ophthalmology, RAF (H), Ely.

Correspondence to: C. M. Jakeman, Department of Ophthalmology, Addenbrookes Hospital, Hills Road, Cambridge CB2 2QQ. 
Hospital all patients meeting these criteria and with cataract sufficient to warrant extraction have been entered into the study.

\section{Preoperative Management}

Immediate preoperative assessment was similar to that for standard cataract patients. Particular attention was paid to the intraocular pressure, anterior and posterior chamber inflammation and degree of iris atrophy. Two eyes were receiving preoperative antiglaucoma therapy. Four eyes were on once or twice daily topical steroid as part of their normal maintenance therapy. No specific attempts were made to adjust these treatments prior to operation.

\section{Operative Procedure}

Preoperative dilation was achieved with Phenylephrine $10 \%$ and Cyclopentolate $1 \%$ 4-6 times in the preceding hour. Surgery was performed under general anaesthesia. The procedure involved a corneal section followed by cystotome anterior capsulotomy ("Canopener" technique) under Healonid ${ }^{\mathrm{R}}$. Following nucleus expression cortical remnants were removed with a manual irrigating and aspirating cannula and Hartmanns solution + 1:1,000,000 adrenaline infusion. A Sinskey style one piece or three piece lens was inserted in the capsular bag. The corneal section was closed with $10 / 0$ nylon sutures. Healonid ${ }^{\mathrm{R}}$ was then exchanged with a proprietary physiological solution.

Subconjunctival Gentamicin $20 \mathrm{mgs}$ and Dexamethasone $1 \mathrm{mg}$ were injected. No iridectomy was performed.

Postoperatively, assessment was made of anterior and posterior segment activity and IOP. Treatment was routinely commenced with four times daily steroid, antibiotic and mydriatic drops unless inflammation was found to be excessive in which case treatment was increased accordingly. Discharge was usually on the second postoperative day. Treatment was continued for a minimum of eight weeks.

\section{Results}

Of our twenty patients $13(65 \%)$ were male and seven $(35 \%)$ were female. We operated on the right eye in $11(55 \%)$ cases and the left eye in nine $(45 \%)$ cases. Three $(15 \%)$ of our cases had bilateral involvement. One had had intracapsular surgery at another hospital 10 years previously, one had had extracapsular surgery without implant and the third has not yet required surgery. These eyes have not been included in the study.

The mean age at presentation was 35.5 years (range 20 to 57 ). The mean age at surgery was 39.9 years (range 22 to 59). The mean duration between presentation and surgery was 4.35 years (range 6 months to 20 years). This includes three cases with documented visual loss from $6 / 6$ to $\mathrm{CF}$ or less within six months.

All cases presented with visual disturbance. Seventeen presented with decreased visual acuity. One presented with monocular diplopia, one with decreased vision associated with floaters and one with a history of a "spiders web" in front of his vision.

Two eyes had had previous surgery. They had required surgery as part of the management of their FHC. One required vitrectomy seven months prior to cataract extraction and one required trabeculectomy ten months prior to cataract extraction.

Preoperative visual acuities and IOPs are summarised in Table I. All Snellen acuities were $6 / 24$ or less immediately prior to surgery. Two patients had raised IOPs prior to surgery despite treatment with topical beta blockers. No additional attempts were made to reduce the pressure further, prior to surgery, in any of the eyes.

There were no operative complications in any of the twenty procedures. A small angle haemorrhage was noted in $15(75 \%)$ eyes. This characteristically occurred shortly after initial entry into the anterior chamber and appeared in the opposite angle, as described by Amsler, ${ }^{17}$ often tracking circumferentially around the angle. The three patients who have required trabeculectomy have all shown angle haemorrhage at both procedures, also as described by Amsler. ${ }^{17}$

In the immediate post operative period four $(20 \%)$ eyes had severe uveitis. These all settled on intensive (1-2 hourly) topical steroid. application over a one to two week period. These eyes did not show an associated elevation in IOP. Two cases settled leaving a partial 
Table I Summary of twenty eyes (IOP-Highest readings given)

\begin{tabular}{rcccccccl}
\hline $\begin{array}{l}\text { Case } \\
\text { No. }\end{array}$ & Age & Sex & $\begin{array}{c}\text { Preop. } \\
\text { Acuity }\end{array}$ & $\begin{array}{c}\text { Postop. } \\
\text { Acuity }\end{array}$ & $\begin{array}{c}\text { Preop. } \\
\text { IOP }\end{array}$ & $\begin{array}{r}\text { Postop. } \\
\text { IOP }\end{array}$ & $\begin{array}{c}\text { Follow up } \\
\text { (Months) }\end{array}$ \\
\hline 1 & 41 & M & $6 / 60$ & $6 / 9$ & 19 & 19 & 73 & Yag capsulotomy 64/12 \\
2 & 34 & M & LP & $6 / 24$ & 38 & 27 & 7 & Trab. 3/12 + Timolol IOP 22 \\
3 & 31 & F & HM & $6 / 6$ & 17 & 11 & 25 & Postop severe uveitis \\
4 & 48 & F & $6 / 36$ & $6 / 9$ & 18 & 14 & 9 & Yag capsulotomy 8/12 \\
5 & 44 & M & $6 / 36$ & $6 / 18$ & 36 & 42 & 8 & Trab. 2/12 IOP 10 \\
6 & 51 & F & HM & $6 / 6$ & 15 & 18 & 11 & \\
7 & 41 & F & CF & $6 / 9$ & 18 & 14 & 9 & \\
8 & 41 & M & HM & $6 / 36$ & 22 & 38 & 60 & Trab. 43/12 IOP 22 \\
9 & 49 & M & CF & $6 / 6$ & 15 & 15 & 7 & Bilateral \\
10 & 44 & F & $6 / 24$ & $6 / 6$ & 16 & 24 & 7 & Bilateral. Yag 5/12. Timolol IOP 17 \\
11 & 27 & M & $6 / 60$ & $6 / 6$ & 18 & 16 & 27 & Postop severe uveitis \\
12 & 27 & M & $6 / 36$ & $6 / 9$ & 10 & 09 & 31 & Bilateral \\
13 & 22 & M & CF & $6 / 5$ & 10 & 11 & 36 & Postop severe uveitis \\
14 & 36 & M & $6 / 60$ & $6 / 9$ & 20 & 16 & 13 & Preop Vitrectomy \\
15 & 59 & M & $6 / 24$ & $6 / 5$ & 14 & 15 & 23 & Postop severe uveitis \\
16 & 34 & M & CF & $6 / 12$ & 14 & 18 & 6 & Capsule thickened \\
17 & 38 & M & $6 / 60$ & $6 / 9$ & 26 & 13 & 9 & Preop Trab. \\
18 & 40 & F & $6 / 60$ & $6 / 6$ & 17 & 15 & 21 & \\
19 & 46 & M & $6 / 24$ & $6 / 6$ & 16 & 12 & 15 & \\
20 & 44 & F & CF & $6 / 6$ & 14 & 15 & 6 & \\
\hline & & & & & & & & \\
\hline
\end{tabular}

pupillary membrane and posterior synechiae formation to the IOL, which were resistant to break down with steroid and mydriatic. These cases were returned to theatre at ten days and two weeks for synechyolysis under local anaesthetic.

There was no residual haemorrhage seen in any of the eyes which had bled at operation. There was no further haemorrhage in any of the eyes.

Intraocular pressures recorded in the first two days postoperatively were all within the normal range, at less than $20 \mathrm{mmHg}$. Even the two eyes with preoperative raised pressure had normal pressures which persisted over at least the first month postoperatively.

The patients have had postoperative follow up for periods between six months and 6 years (mean 20.2 months). Within this period four eyes have shown evidence of raised pressure.

Two of these eyes had shown elevated pressures prior to surgery. They have subsequently required trabeculectomy at two and three months with so far successful outcome although one requires topical timolol to maintain the pressure below $22 \mathrm{mmHg}$. One eye has required trabeculectomy at 43 months. Unfortunately the pressure in this eye is still not adequately controlled and there has been some central field loss owing to glaucoma.
One eye has had minimally elevated pressure since four months post-operatively but is so far controlled on timolol. The eye with preoperative trabeculectomy has continued to maintain a satisfactory pressure.

The postoperative visual acuities and visual improvement are summarised in Table I and Figure 1. Three eyes have required Yag capsulotomy for thickened posterior capsules at five months, eight months and 64 months.

\section{Discussion}

The implantation of intraocular lenses follow-

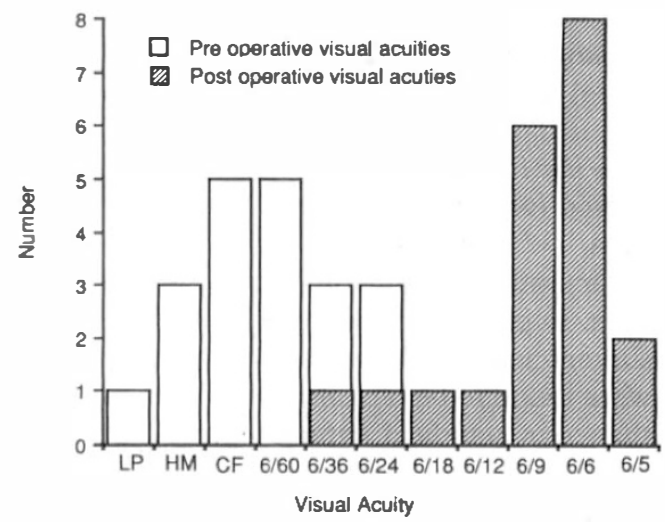

Fig. 1 Comparison of Preoperative and Postoperative Visual Acuities 
ing cataract surgery into eyes with chronic uveitis is controversial. The recent study by Foster ${ }^{18}$ has shown that the results from surgery can be successful. He stresses the need to defer surgery until inflammation has been eliminated and also that the visual outcome can be disappointing due to associated macular problems.

We have long felt that Fuchs' cyclitis despite being a chronic 'uveitis' represents a less aggressive disease and that these eyes should tolerate surgery and lens implantation more readily. The lack of associated macular problems should also provide a more reliable outcome.

Unfortunately early results from cataract surgery in FHC have been contradictory and somewhat disappointing. Ward and $\mathrm{Hart}^{8}$ describe nine case histories of complicated cataract extraction. They identify glaucoma, post operative intraocular haemorrhage and vitreous opacification as major problems. Norn ${ }^{19}$ describes four of twenty-three cases, following surgery, requiring enucleation owing to complications. More recently with improved techniques Mills and Rosen ${ }^{9}$, Mooney and O'Conner ${ }^{10}$ and Turut $^{11}$ have reported more encouraging results following combined cataract extraction and lens implantation using an anterior chamber, iris fixated or iridocapsular fixated lens. They still identified pressure elevation and vitreous opacity as problems.

When commencing this study we hoped that the use of more modern techniques and particularly the use of a posterior chamber lens would result in a lower incidence of complications and still maintain good long term visual function. In view of the abnormal iris and iris/angle vasculature in FHC we believe that iris fixated, iridocapsular, or angle supported implants should not be used in these eyes.

Operation was uncomplicated in all twenty eyes. In particular the two eyes with previous surgery and the two eyes with raised pressure were found to be technically no more difficult than our standard cataract procedure. The small angle haemorrhage in 15 cases caused no additional problems and with the aid of Healonid $^{\mathrm{R}}$ in the anterior chamber did not obstruct our view. There was no relation between the appearance of haemorrhage and postoperative uveitis or raised pressure. In all cases the haemorrhage had cleared by the following morning.

In the immediate postoperative period no eyes had elevated intra-ocular pressures. Four eyes had marked anterior uveitis but we could find no correlation between this finding and our preoperative or operative findings. All four eyes were treated with intensive topical steroid and mydriatic and eventually settled without additional therapy. Two eyes were left with partial pupil membranes and posterior synechiae to the IOL. Although both of these cases were returned to theatre for synechyolysis, in retrospect, this was probably not required. Both eyes now have $6 / 6$ or better vision and require no maintenance therapy.

Raised intra-ocular pressure has caused problems in five eyes $(25 \%)$ at some stage during our study. In three eyes the pressure was known to be elevated prior to cataract surgery, and of these, two were responding poorly to treatment prior to surgery. It should be remembered that the underlying condition has a high risk of the development of glaucoma with Liesegang ${ }^{4}$ reporting its development in $59 \%$ of his cases during his long term follow up. The glaucoma associated with FHC is also known to respond poorly to both medical and surgical management. ${ }^{4}$

Three of the eyes in our series have had visual problems related to vitreous opacity. This is another common complication of Fuchs cyclitis and is used as a diagnostic criteria. ${ }^{7,17}$ It does not seem to be causally related to cataract extraction with lens implantation. However, the dense lens opacity often obscures its presence preoperatively. We certainly have not witnessed any change in vitreous opacity directly related to surgery. The one case in which pars plana vitrectomy preceded cataract extraction has had an excellent visual outcome (6/9) with no obvious increased risk of complications at cataract surgery.

Comparison of visual acuity pre and postoperatively shows all cases to have gained some visual improvement from cataract extraction. Sixteen eyes $(80 \%)$ have vision equal to or better than $6 / 9$. One eye with $6 / 12$ vision has slight posterior capsule thickening and vision should be improved by Yag capsu- 
lotomy. The remaining eyes have poor vision related to a combination of vitreous opacity, glaucomatous field loss and macular oedema.

Although some of our cases have only short term follow up, in the seven cases with two or more years review there seems to be no evidence of long term problems, attributable to the cataract surgery, developing in these eyes. Only one of these cases has lost vision due to poorly controlled glaucoma. With this one exception there is no evidence of increasing pressure, increasing uveitis, corneal decompensation or declining vision in these eyes.

\section{Conclusions}

We conclude that cataract extraction and posterior chamber lens implantation in Fuchs cyclitis is a safe procedure. Age, initial visual acuity and duration of symptoms seem unrelated to final vision achieved. The operation is no more complicated than a standard cataract extraction with IOL. Obviously when operating on such a young age group special care should be taken and we recommend the routine use of Healonid ${ }^{\mathrm{R}}$. Although our study showed no advantage with lens style, on theoretical grounds, we recommend the use of a one piece PMMA lens with capsular bag fixation. The use of hydrophilic or heparin coated lenses should also be considered.

We consider that the few complications we encountered cannot definitely be attributed to the operative procedure. They probably represent the natural history of the underlying condition.

We do not consider glaucoma a contraindication to surgery. Careful thought should be given to the likely outcome, and the patient should be given a guarded prognosis, before surgery is undertaken.

\footnotetext{
References

${ }^{1}$ Schlaegel TF Jr: Differential diagnosis of uveitis. Ophthalmol Digest 1973, 35: 34.
}

${ }^{2}$ Kijlstra A, Rothova A, Baarsma GS, Zaal MJ, Fortuin ME, Schweitzer C, Glasius E, de Jong PT: Computer registration of uveitis patients. Doc Ophthalmol 1987, 67 (1-2): 139-43.

${ }^{3}$ Perkins ES: Heterochromic Uveitis. Trans Ophthalmol Soc UK 1961, 81: 53-66.

${ }^{4}$ Liesegang TJ: Clinical features and prognosis in Fuchs' Uveitis Syndrome. Arch Ophthalmol 1982, 100: 1622-26.

${ }^{5}$ O'Connor GR: Heterochromic Iridocyclitis. Trans Ophthalmol Soc UK 1985, 104: 219-31.

${ }^{6}$ Smith RE and O'Connor GR: Cataract extraction in Fuchs' Syndrome. Arch Ophthalmol 1974, 91: 39-41.

${ }^{7}$ Fraceschetti A: Heterochromic Cyclitis (Fuchs' Syndrome). Am J Ophthalmol 1955, 39: 50-8.

${ }^{8}$ Ward DM and Hart CT: Complicated cataract extraction in Fuchs' Heterochromic uveitis. $\mathrm{Br} J$ Ophthalmol 1967, 51: 530-8.

${ }^{9}$ Mills KB and Rosen ES: Intra-ocular lens implantation following cataract extraction in Fuchs' Heterochromic Uveitis. Ophthalmic Surgery 1982, 13: 467-9.

${ }^{10}$ Mooney D and O'Connor M: Intraocular lenses in Fuchs' Heterochromic Cyclitis. Trans Ophthalmol Soc UK 1980, 100: 510.

${ }^{11}$ Turut P, Malthieu C, Malthieu D, Pfaelzer I: Cristallin Artificiel et Syndrome De Fuchs'. Bull Soc Ophthalmol Fr 1984, 10: 1147-53.

${ }^{12}$ Loewenfeld IE and Thompson HA: Fuchs' Heterochromic Cyclitis. A critical review of the literature. Surv Ophthalmol 1973, 17: 394-457.

${ }^{13}$ Berger BB, Tessler HH, Kottow MH: Anterior segment ischaemia in Fuchs' Heterochromic Cyclitis. Arch Ophthalmol 1980, 98: 499-.

${ }^{14}$ Brooks AMV, Grant G, Gilles WE: Changes in the iris vasculature and corneal endothelium in chronic cyclitis. Austr New Z J Ophthalmol 1986, 14: 189-97.

${ }^{15}$ Sugar HS: Heterochromia iridis with special consideration of its relation to cyclitic disease. $A m \mathrm{~J}$ Ophthalmol 1965, 69: 1-18.

${ }^{16}$ Amsler M and Verrey F: Heterochromie de Fuchs et fragilite vasculaire. Ophthalmologica, Basel 1946, 111: 177-81.

${ }^{17}$ Fuchs E: Ueber Komplikationen der Heterochromie. Z. Augenheilk 1906, 15: 191-212.

${ }^{18}$ Foster CS, Fong LP, Singh G: Cataract surgery and intraocular lens implantation in patients with uveitis. Ophthalmology 1989, 96: 281-8.

${ }^{19}$ Norn MS: cataract extraction in Fuchs' Heterochromia Acta Ophthalmol 1968, 46: 685-99. 